\title{
UNCERTAINTY PRINCIPLES FOR THE AFFINE GROUP
}

\author{
Hans Martin ReimanN
}

Dedicated to Professor Bogdan Bojarski on the occasion of his 75 th birthday

\begin{abstract}
The Lie algebra of the affine group is generated by two operators $A$ and $B$ satisfying the commutator rule $[A, B]=B$. A version of the uncertainty principle is designed such that - in the time domain - the extremal functions are real valued. The uncertainty inequality naturally contains a parameter. In the application the wavelet transform based on the extremal functions gives a model for the first stage of the hearing perception in the inner ear (the cochlea). The parameter in the uncertainty inequality is associated to the position along the cochlea.
\end{abstract}

Keywords: Uncertainty principle, affine group, cochlea.

\section{Introduction}

The quest to understand signal processing as it is done by the human ear lies at the origin of this study. Physiological experiments reveal the importance of the affine group $\Gamma$. Invariance principles naturally guide us to the action of the circle group $S$, the distinguished one parameter transformation group which commutes with the action of the affine group. The main result is the basic uncertainty principle for $\Gamma \times S$ :

$$
\frac{\nu^{2}}{4} \leqslant \tau^{2}\left(-\frac{1}{4}+\sigma^{2}\right)
$$

It is a way of expressing that the frequency content of a signal $\left(\tau^{2}\right)$ and its temporal variation $\left(\sigma^{2}\right)$ cannot simultaneously be small. The parameter $\nu$ which figures in this inequality is the momentum of the frequency distribution.

The Heisenberg uncertainty principle tells us, that time localization and specification of the frequency content of an acoustic signal cannot be achieved simultaneously with arbitrary precision. This is of central importance in signal processing and many studies are devoted to this topic (see the standard texts in signal processing, e.g. Mallat 1998).

The uncertainty principle for scale by Cohen (1993) is at the beginning of a new development. The idea was taken up in auditory modeling by Irino and

Mathematics Subject Classification: $43 \mathrm{~A} 80$ 
Patterson (1997). It led to their gammachirp auditory filter, which models hearing perception. Indeed, their work is a source of inspiration for the present paper.

In 1946, Gabor defined elementary time-frequency atoms as extremals for the Heisenberg uncertainty principle and stressed the importance of localized timefrequency signal processing. The present study reveals, that the ear is sensible to the elementary time-frequency atoms which arise as extremals in the uncertainty principle for the affine group.

In contrast to the classical situation, the uncertainty principle for the affine group contains an additional parameter. In the inner ear this parameter is associated with the place along the cochlea and in signal processing it is interpreted as a frequency momentum.

Heisenberg's uncertainty principle has its origin in quantum physics. The underlying function space is a space of complex valued functions. The space of acoustic signals however consist of real valued functions. This is the natural setting for the uncertainty principle for the affine group. The results can directly be applied to signal processing. Of particular interest are the extremal functions, the time-frequency atoms, which - needless to say - are real valued functions.

In the last section we note that Heisenberg's uncertainty principle can also be formulated in this framework.

\section{The symmetry group on the space of acoustic signals}

Acoustic signals can be described by pressure fluctuations $f(t)$ in the time variable $t$. The energy of the signal is $\int_{-\infty}^{\infty}|f(t)|^{2} d t$. The space of acoustic signals is therefore $L^{2}(\mathbb{R}, \mathbb{R})$, the Hilbert space of real valued square integrable functions.

The affine group

$$
\begin{aligned}
\Gamma & =\{(a, b): a>0, b \in \mathbb{R}\} \\
& =\{(a, b): t \rightarrow a t+b, a>0, b \in \mathbb{R}\}
\end{aligned}
$$

with group law

$$
(a, b)\left(a^{\prime}, b^{\prime}\right)=\left(a a^{\prime}, a b^{\prime}+b\right)
$$

acts on $L^{2}(\mathbb{R}, \mathbb{R})$. Translations and dilations induce actions on $L^{2}(\mathbb{R}, \mathbb{R})$. The translations

$$
\tau(b): t \rightarrow t+b
$$

describe the time shift. This action is quite natural and reflects time invariance. The dilations

$$
\delta(a): t \rightarrow a t
$$

however enter the picture only at the level of the hearing process. They reflect that the ear recognizes a sound independently of its basic pitch. 
The induced actions on $L^{2}(\mathbb{R}, \mathbb{R})$ are denoted with the same symbols:

$$
\begin{aligned}
\delta(a) f(t) & =\frac{1}{\sqrt{a}} f\left(\frac{t}{a}\right) \\
\tau(b) f(t) & =f(t-b)
\end{aligned}
$$

The factor $\frac{1}{\sqrt{a}}$ is introduced in order that the action is orthogonal (unitary). We then set

$$
\rho(a, b) f(t)=\frac{1}{\sqrt{a}} f\left(\frac{t-b}{a}\right)=\tau(b) \delta(a) f(t)
$$

The Fourier transform $f$ intertwines the unitary representation $\left(\rho, L^{2}(\mathbb{R}, \mathbb{R})\right)$ of $\Gamma$ with the representation $\left(\hat{\rho}, L_{\text {sym }}^{2}\right)$, where

$$
\begin{aligned}
L_{\mathrm{sym}}^{2} & =\left\{\hat{f} \in L^{2}(\mathbb{R}, \mathbb{C}): \hat{f}(\omega)=\overline{\hat{f}(-\omega)}\right\} \\
\hat{\delta}(a) \hat{f}(\omega) & =\sqrt{a} \hat{f}(a \omega) \\
\hat{\tau}(b) \hat{f}(\omega) & =e^{-i b \omega} \hat{f}(\omega) \\
\hat{\rho}(a, b) & =\hat{\tau}(b) \hat{\delta}(a) \hat{f}(\omega)=e^{-i \omega b} \sqrt{a} \hat{f}(a \omega)
\end{aligned}
$$

It should be noted that $L_{\text {sym }}^{2}$ is a real subspace of the complex Hilbert space $L^{2}(\mathbb{R}, \mathbb{C})$. It is a real Hilbert space, the scalar product being given as the restriction of the Hermitian scalars product in $L^{2}(\mathbb{R}, \mathbb{C})$ to $L_{\text {sym }}^{2}$.

The multiplicative group $S=\left\{\varepsilon(\varphi)=e^{i \varphi}: \varphi \in \mathbb{R}\right\}$ of complex numbers of absolute value one acts in a natural way on $L_{\text {sym }}^{2}$ :

$$
\begin{aligned}
\hat{\varepsilon}(\varphi) \hat{f}(\omega) & =e^{-i \varphi \operatorname{sign}(\omega)} \hat{f}(\omega) \\
& =\cos (\varphi) \hat{f}(\omega)-i \sin (\varphi) \operatorname{sign}(\omega) \hat{f}(\omega)
\end{aligned}
$$

The function $-i \operatorname{sign}(\omega) \hat{f}(\omega)$ is the Fourier transform of the Hilbert transform $H f$ of $f$ :

$$
\begin{aligned}
& H f(t)=\frac{1}{\pi} \lim _{\varepsilon \rightarrow 0} \int_{|s| \geqslant \varepsilon} \frac{f(s)}{t-s} \mathrm{ds}, \\
& -i \operatorname{sign}(\omega) \hat{f}(\omega)=\hat{H} f(\omega)(g)
\end{aligned}
$$

The action of $S$ on $L^{2}(\mathbb{R}, \mathbb{R})$ is therefore

$$
\varepsilon(\varphi) f(t)=\cos (\varphi) f(t)+\sin (\varphi) H f(t)
$$

It should be noted, that the S-action commutes with the $\Gamma$-action. The representations $\rho$ and $\hat{\rho}$ of $\Gamma$ can thus be extended to representations of $\Gamma \times S$

$$
\begin{aligned}
\rho(a, b, \varphi) & =\varepsilon(\varphi) \rho(a, b) \\
\hat{\rho}(a, b, \varphi) & =\hat{\varepsilon}(\varphi) \hat{\rho}(a, b)
\end{aligned}
$$

The symmetry group on the space $L^{2}(\mathbb{R}, \mathbb{R})$ of signals is therefore the group $\Gamma \times S$. The one parameter group $S$ of transformation is distinguished by its invariance properties. This is a well known fact in harmonic analysis. 
Proposition 2.1. If $G$ is a uniformly bounded one parameter group of linear transformations of $L^{2}(\mathbb{R}, \mathbb{R})$, which commutes with translations and dilations, then $G$ is the transformation group $S-$ up to scaling.

Proof. In fact, translation invariant linear operators $T$ of $L^{2}(\mathbb{R})$ are multiplier operators (see e.g. [S] p. 28). There exists a function $m \in L^{\infty}(\mathbb{R})$ such that

$$
(T f)^{\wedge}(\omega)=m(\omega) \hat{f}(\omega) \quad \text { a.e. }
$$

for all $f \in L^{2}(\mathbb{R})$. If $T$ is dilation invariant then the multiplier satisfies

$$
m(c \omega)=m(\omega), \quad c>0
$$

Hence there exist constants $a, b$ such that

$$
m(\omega)= \begin{cases}a, & \omega>0 \\ b, & \omega<0\end{cases}
$$

If $T$ preserves real valued functions, then $b=\bar{a}$. Writing $a=r e^{-i \varphi}$ the multiplier is

$$
m(\omega)=r e^{-i \varphi \operatorname{sign}(\omega)}
$$

A uniformly bounded one parameter group $G$ of such operators must then be the transformation group $S$-up to a scaling of the parameter $\varphi$.

The role of the $S$-action in hearing has not been clarified yet. It is my conjecture, that the hearing process carefully controls the S-action. This question is related to the problem of "hearing phases". According to Helmholtz $[\mathrm{H}]$ "The quality of the musical portion of a composed tone depends solely on the number and relative strength of its partial simple tones, and in no respect on their differences of phases".

It has been known for some time that the statement of Helmholtz is not valid for sounds composed of many harmonics, see e.g. R.Patterson $[\mathrm{P}]$ and the references given in this paper.

There are two further heuristic reasons why the S-action should be preserved.

1. The Hilbert transform, which is the action $\varepsilon\left(\frac{\pi}{2}\right)$, reverses symmetries. It maps even functions into odd functions and vice versa. Hearing is sensible to symmetries.

2. The Hilbert transform does not preserve functions of compact support. If the hearing process would not preserve the S-action it could not control the support and hence the timing of the acoustic signals.

The representations $\left(\rho, L^{2}(\mathbb{R}, \mathbb{R})\right)$ and $\left(\hat{\rho}, L_{\text {sym }}^{2}\right)$ of $\Gamma \times S$ induce representations of the Lie Algebra. The infinitesimal generators of the one parameter groups 
$\delta\left(e^{s}\right), \tau(b)$ and $\varepsilon(\varphi)$ are

$$
\begin{aligned}
& A f(t)=\left.\frac{d}{d s}\right|_{s=0} \delta\left(e^{s}\right) f(t)=\left.\frac{d}{d s}\right|_{s=0} e^{-s / 2} f\left(e^{-s} t\right)=-\frac{1}{2} f(t)-t \frac{d f}{d t}(t), \\
& B f(t)=\left.\frac{d}{d b}\right|_{b=0} \tau(b) f(t)=\left.\frac{d}{d b}\right|_{b=0} f(t-b)=-\frac{d f}{d t}(t), \\
& H f(t)=\left.\frac{d}{d \varphi}\right|_{\varphi=0}(\cos (\varphi) f(t)+\sin (\varphi) H f(t))=H f(t) .
\end{aligned}
$$

In the Fourier transform picture we have

$$
\begin{aligned}
& \hat{A} \hat{f}(\omega)=\left.\frac{d}{d s}\right|_{s=0} \hat{\delta}\left(e^{s}\right) \hat{f}(\omega)=\left.\frac{d}{d s}\right|_{s=0} e^{s / 2} \hat{f}\left(e^{s} \omega\right)=\frac{1}{2} \hat{f}(\omega)+\omega \frac{d \hat{f}}{d \omega}(\omega), \\
& \hat{B} \hat{f}(\omega)=\left.\frac{d}{d b}\right|_{b=0} e^{-i b \omega} \hat{f}(\omega)=-i \omega \hat{f}(\omega), \\
& \hat{H} \hat{f}(\omega)=\left.\frac{d}{d \varphi}\right|_{\varphi=0} e^{-i \varphi \operatorname{sign}(\omega)} \hat{f}(\omega)=-i \operatorname{sign}(\omega) \hat{f}(\omega)
\end{aligned}
$$

The only non-vanishing brackets are

$$
\begin{array}{ll}
{[A, B]=B} & \text { for the representation } \rho, \\
{[\hat{A}, \hat{B}]=\hat{B}} & \text { for the representation } \hat{\rho} .
\end{array}
$$

Since the representation is unitary, the infinitesimal generators are skew adjoint and the domain of definition is dense in the Hilbert space (see [T], Theorem 1.5, p.6).

There is another way of looking at the representations $\rho$ and $\hat{\rho}$. The Hilbert transform $H$ satisfies $H^{2}=-I$. It therefore defines a complex structure on $L^{2}(\mathbb{R}, \mathbb{R})$ (and similarly for $\hat{H}$ on $L_{\text {sym }}^{2}$ ). Since $\delta(a)$ and $\tau(b)$ commute with $H$ we can consider $\rho$ as a complex unitary representation on $L^{2}(\mathbb{R}, \mathbb{R})$ equipped with the complex structure $H$. The action $\varepsilon(\varphi)$ is then the natural action of the group $S$ of complex numbers of absolute value one.

\section{The basic uncertainty inequality}

The uncertainty inequality for $\Gamma \times S$ is based on the following calculation:

For all $\lambda, \mu \in \mathbb{R}$

$$
\begin{aligned}
0 & \leqslant\|(\lambda A+\mu H B) f\|^{2}=\lambda^{2}\|A f\|^{2}+\lambda \mu(A f, H B f)+\lambda \mu(H B f, A f)+\mu^{2}\|H B f\| \\
& =\lambda^{2}\|A f\|^{2}-\lambda \mu(f,[A, H B] f)+\mu^{2}\|H B f\|^{2}
\end{aligned}
$$

With $[A, H B]=H B$ it follows that

$$
|(f, H B f)| \leqslant 2\|A f\|\|H B f\|
$$


Observe that the scalar products $(A f, H B f)$ and $(f, H B f)$ are finite if $A f$ and $B f$ are in $L^{2}(\mathbb{R}, \mathbb{C})$. The inequality holds trivially, if either $\|A f\|=\infty$ or $\|B f\|=\infty$. Equality is reached if and only if

$$
(\lambda A+\mu H B) f=0
$$

for some $\lambda, \mu \in \mathbb{R}^{2},(\lambda, \mu) \neq(0,0)$. form

If $(\lambda A+\mu H B) f=0$ for some $(\lambda, \mu) \in \mathbb{R}^{2}$, then the non-negative quadratic

$$
\lambda^{2}\|A f\|^{2}-\lambda \mu(f, H B f)+\mu^{2}\|H B f\|^{2}
$$

vanishes for these values $\lambda, \mu$. Therefore the discriminant has to vanish:

$$
(f, H B f)^{2}-4\|A f\|^{2}\|H B f\|^{2}=0 .
$$

Conversely, if equality holds for $f(\neq 0)$, the discriminant of the quadratic form vanishes. There exist then $(\lambda, \mu) \in \mathbb{R}^{2},(\lambda, \mu) \neq(0,0)$ such that

$$
\lambda^{2}\|A f\|^{2}-\lambda \mu(f, H B f)+\mu^{2}\|H B f\|^{2}=0
$$

for these values $\lambda, \mu$ one must have

$$
\|(\lambda A+\mu H B) f\|=0
$$

and hence

$$
(\lambda A+\mu H B) f=0 .
$$

In the deduction of the uncertainty inequality, $H B$ can be replaced by $H B-\eta I$, since both $H B$ and $I$ are selfadjoint. The skew adjoint operator $A$ can be replaced by $A-\beta B$, since $B$ commutes with $H B$. The basic uncertainty inequality then takes the form

$$
|(f, H B f)| \leqslant 2\|(A-\beta B) f\|\|(H B-\eta I) f\| .
$$

Set

$$
\nu\|f\|^{2}=-(f, H B f)=-(\hat{f}, \hat{H} \hat{B} \hat{f})=\int_{-\infty}^{\infty}|\omega||\hat{f}(\omega)|^{2} \mathrm{~d} \omega .
$$

Then $\nu$ is the expectation value of the modulus of the frequency of the signal. As such it is a positive real number (and the operator $-H B$ is positive).

Observe next that the norm $\|(H B-\eta I) f\|$ is minimal if $\eta=-\nu$. The expression $\|(A-\beta B) f\|$ is minimal if

$$
\beta=\frac{\operatorname{Re}(A f, B f)}{\|B f\|^{2}}
$$


In what follows, $\beta$ is taken to be this number. For $\operatorname{Re}(A f, B f)$ one calculates

$$
\begin{aligned}
\operatorname{Re}(A f, B f) & =\operatorname{Re} \int_{-\infty}^{\infty}\left(-\frac{f(t)}{2}-t \frac{d f}{d t}(t)\right)\left(-\frac{d \bar{f}}{d t}(t)\right) d t \\
& =\int_{-\infty}^{\infty} t\left|\frac{d f}{d t}(t)\right|^{2} d t .
\end{aligned}
$$

Therefore $\beta$ is the expectation value for the position of the derivative of $f$

$$
\beta=\frac{\int_{-\infty}^{\infty} t\left|\frac{d f}{d t}(t)\right|^{2} d t}{\int_{-\infty}^{\infty}\left|\frac{d f}{d t}(t)\right|^{2} d t}
$$

The precise uncertainty inequality then takes the form

$$
\nu\|f\|^{2} \leqslant 2\|(A-\beta B) f\|\|(H B+\nu) f\|
$$

The mean deviation from the expectation value $\nu$ for the modulus of the frequency is

$$
\tau^{2}=\frac{\|(H B+\nu I) \hat{f}\|^{2}}{\|\hat{f}\|^{2}}=\frac{1}{\|f\|^{2}} \int_{-\infty}^{\infty}(|\omega|-\nu)^{2}|\hat{f}(\omega)|^{2} d \omega
$$

The other factor in the inequality has a similar interpretation.

$$
\begin{aligned}
\|(A-\beta B) f\|^{2} & =\|A f\|^{2}-2 \beta 2 R e(A f, B f)+\beta^{2}\|B f\|^{2} \\
& =\frac{1}{4}\|f\|^{2}+\operatorname{Re}\left(f, t \frac{d f}{d t}\right)+\left\|t \frac{d f}{d t}\right\|^{2}-2 \beta\left(t \frac{d f}{d t}, \frac{d f}{d t}\right)+\beta^{2}\left\|\frac{d f}{d t}\right\|^{2} \\
& =-\frac{1}{4}\|f\|^{2}+\int_{-\infty}^{\infty}(t-\beta)^{2}\left|\frac{d f}{d t}(t)\right|^{2} d t \\
& =-\frac{1}{4}\|f\|^{2}+\sigma^{2}\|f\|^{2}
\end{aligned}
$$

The quantity $\sigma^{2}$ is the mean deviation from the expectation value $\beta$ related to the square norm of $f$ (and not to the square norm of $\frac{d f}{d t}$ ).

$$
\sigma^{2}=\frac{1}{\|f\|^{2}} \int_{-\infty}^{\infty}(t-\beta)^{2}\left|\frac{d f}{d t}(t)\right|^{2} d t
$$

Theorem 3.1 (uncertainty principle). For any $f \in L^{2}(\mathbb{R}, \mathbb{C})$ with $A f$ and $B f$ in $L^{2}(\mathbb{R}, \mathbb{C})$ set

$$
\beta=\frac{\int_{-\infty}^{\infty} t\left|\frac{d f}{d t}(t)\right|^{2} d t}{\int_{-\infty}^{\infty}\left|\frac{d f}{d t}(t)\right|^{2} d t},
$$




$$
\begin{gathered}
\nu=\frac{1}{\|f\|^{2}} \int_{-\infty}^{\infty}|\omega||\hat{f}(\omega)|^{2} d \omega \\
\sigma^{2}=\frac{1}{\|f\|^{2}} \int_{-\infty}^{\infty}(t-\beta)^{2}\left|\frac{d f}{d t}(t)\right|^{2} d t \\
\tau^{2}=\frac{1}{\|f\|^{2}} \int_{-\infty}^{\infty}(|\omega|-\nu)^{2}|\hat{f}(\omega)|^{2} d \omega .
\end{gathered}
$$

Then the inequality

$$
\frac{\nu^{2}}{4} \leqslant\left(-\frac{1}{4}+\sigma^{2}\right) \tau^{2}
$$

holds.

The quantities $\beta, \nu, \tau$ and $\sigma$ transform as follows under the representation $\rho$. Set $f_{a, b}=\rho(a, b) f, \hat{f}(a, b)=\hat{\rho}(a, b) \hat{f}$. Then

$$
\begin{gathered}
\beta\left(f_{a, b}\right)=\frac{\int_{-\infty}^{\infty} t\left|\frac{d f_{a, b}}{d t}(t)\right|^{2} d t}{\int_{-\infty}^{\infty}\left|\frac{d f_{a, b}}{d t}(t)\right|^{2} d t}=a \beta(f)+b, \\
\nu\left(f_{a, b}\right)=\frac{1}{\left\|\hat{f_{a, b}}\right\|^{2}} \int_{-\infty}^{\infty}\left|\omega \| \hat{f_{a, b}}(\omega)\right|^{2} d \omega=a^{-1} \nu(f), \\
\sigma^{2}\left(f_{a, b}\right)=\frac{1}{\left\|f_{a, b}\right\|^{2}} \int_{-\infty}^{\infty}\left(t-\beta\left(f f_{a, b}\right)\right)^{2}\left|\frac{d f_{a, b}}{d t}(t)\right|^{2} d t=\sigma^{2}(f), \\
\tau^{2}\left(f_{a, b}\right)=\frac{1}{\| \hat{f_{a, b} \|^{2}}} \int_{-\infty}^{\infty}\left(|\omega|-\nu\left(\hat{f_{a, b}}\right)\right)^{2}\left|\hat{f_{a, b}}(\omega)\right|^{2} d \omega=a^{-2} \tau^{2}(f)
\end{gathered}
$$

A function $f$ for which equality holds in the uncertainty principle will be called an extremal function. The reasoning used at the beginning of this section shows that $f$ is extremal if and only if there exist $\lambda, \mu \in \mathbb{R}$ such that

$$
\lambda(A-\beta B) f+\mu(H B-\eta I) f=0
$$

If $f \in L^{2}(\mathbb{R}, \mathbb{R})$ is a solution of this equation, then this forces $\beta$ to be the expectation value for the position of $\frac{d f}{d t}$ and $-\eta=\nu$ the expectation value for the modulus of the frequency of $\hat{f}$ (as defined above). 
Fortunately, the Fourier transforms $\hat{f}$ of the extremal functions can be determined explicitly: in the equality

$$
\lambda(\hat{A}-\beta \hat{B}) \hat{f}+\mu(\hat{H} \hat{B}+\nu I) \hat{f}=0
$$

we specify the operators and set $\hat{f}=h, \frac{\mu}{\lambda}=-\kappa$ :

$$
\left(\frac{1}{2}+\omega \frac{d}{d \omega}+i \beta \omega\right) h=-\kappa(|\omega|-\nu) h
$$

This differential equation can be separated:

$$
\frac{\frac{d h}{d \omega}}{h}=-\kappa \operatorname{sign}(\omega)-i \beta+\left(\kappa \nu-\frac{1}{2}\right) \frac{1}{\omega}
$$

The solutions in $L_{\text {sym }}^{2}$ of this equation are

$$
h(\omega)=c e^{-i \varepsilon \operatorname{sign}(\omega)-i \beta \omega} e^{-\kappa|\omega|}|\omega|^{\kappa \nu-\frac{1}{2}}
$$

where $c$ and $\varepsilon$ are arbitrary real constants. The parameters are $\nu=\nu(h), \beta=\beta(h)$ and $\kappa=\kappa(h)$. Both $\nu$ and $\kappa$ must be positive. The parameter $\kappa$ can be calculated from the defining equality:

$$
\begin{aligned}
\|(A-\beta B) h\|^{2} & =\kappa^{2}\|(H B+\nu I) h\|^{2}, \\
-\frac{1}{4}+\sigma^{2} & =\kappa^{2} \tau^{2} .
\end{aligned}
$$

Since $h$ is extremal, the equality

$$
-\frac{1}{4}+\sigma^{2}=\frac{\nu^{2}}{4 \tau^{2}}
$$

has to hold.

Therefore

$$
\kappa=\frac{\nu}{2 \tau^{2}}=\frac{2}{\nu}\left(-\frac{1}{4}+\sigma^{2}\right)
$$

These functions are in $L_{\text {sym }}^{2}$ provided that $\kappa$ and $\nu$ both are positive.

Note that $h$ may not be continuous at 0 . Still it is a solution of the differential equation. The differential operator $\omega \frac{d}{d \omega}$ can be applied to $h$ in the distributional sense.

As such $\omega \frac{d}{d \omega} h$ is the temperate distribution defined by

$$
\omega \frac{d}{d \omega} h(\varphi)=-\int h(\omega) \frac{d}{d \omega}(\omega \varphi(\omega)) d \omega
$$

for any test function $\varphi$.

Another possibility is to interpret the differential operator as a Lie derivative:

$$
\left(\omega \frac{d}{d \omega} h\right)(\omega)=\left.\frac{d}{d s}\right|_{s=0} h\left(e^{s} \omega\right)
$$

Both variants show that $h$ is a solution in $L_{\mathrm{sym}}^{2}$ of the differential equation. 
Proposition 3.1. The set $E$ of extremal functions for the uncertainty inequality that are in $L^{2}(\mathbb{R}, \mathbb{R})$ is invariant under the action $\rho$ of $\Gamma \times S$.

Proof. It suffices to show that the set $\hat{E}$ of Fourier transforms of extremal functions is invariant under the action $\hat{\rho}$ of $\Gamma \times S$. But this can readily be seen from the explicit form of the solutions:

$$
\hat{\rho}(a) h(\omega)=c a^{\kappa \nu} e^{-i \varepsilon \operatorname{sign}(\omega)} e^{-\kappa a|\omega|-i \beta a \omega}|\omega|^{\kappa a \nu a^{-1}-\frac{1}{2}}
$$

Under this action the parameters change as

$$
(\kappa, \nu, \beta, \varepsilon) \rightarrow\left(\kappa a, \nu a^{-1}, \beta a, \varepsilon\right)
$$

similarly

$$
\hat{\tau}(b) \hat{\varepsilon}(\varphi) h(\omega)=c e^{-i(\varepsilon+\varphi) \operatorname{sign}(\omega)} e^{-\kappa|\omega|-i(\beta+b) \omega}|\omega|^{\kappa \nu-\frac{1}{2}}
$$

with parameter change

$$
(\kappa, \nu, \beta, \varepsilon) \rightarrow(\kappa, \nu, \beta+b, \varepsilon+\varphi) .
$$

Extension. Starting from the inequality

$$
|(f, H B f)| \leqslant 2\|A f\|\|H B f\|
$$

it is possible to replace $A$ by $A-\alpha H-\beta B$ instead of by $A-\beta B$. The resulting uncertainty inequality

$$
\nu\|f\|^{2} \leqslant 2\|(A-\alpha H-\beta B) f\|\|(H B+\nu I) f\|
$$

still displays the full invariance under $\Gamma$.

The condition for minimality of $\alpha$ and $\beta$ is now a linear system of two equations:

$$
\begin{gathered}
\alpha\|f\|^{2}-\beta(f, H B f)+(f, H A f)=0, \\
-\alpha(f, H B f)+\beta\|B f\|^{2}-\operatorname{Re}(A f, B f)=0 .
\end{gathered}
$$

The Fourier transforms of the extremal functions then satisfy the differential equation

$$
\lambda(\hat{A}-\alpha \hat{H}-\beta \hat{B}) \hat{f}+\mu(\hat{H} \hat{B}+\nu I) \hat{f}=0
$$

with $\alpha$ and $\beta$ the solutions of the above system. A direct calculation shows that they are of the form

$$
h(\omega)=c e^{-i \varepsilon \operatorname{sign}(\omega)-i \alpha \operatorname{sign}(\omega) \log |\omega|-i \beta \omega} e^{-\kappa|\omega|}|\omega|^{\kappa \nu-\frac{1}{2}}
$$




\section{The basilar membrane filter}

In the inner ear the acoustic signals $f$ are transformed into fluid waves. They travel along the basilar membrane, where the hair cells are located. These cells convert the movement of the basilar membrane into nerve impulses.

For a fixed position $x$ along the basilar membrane the response to a sinusoidal input increases with the frequency $\omega$ up to a critical frequency $\omega_{x}$, the so called resonant frequency. If the frequency is increased beyond $\omega_{x}$, the response quickly drops to zero. The function which assigns to $x$ the resonant frequency $\omega_{x}$ is called the frequency position function. In a first approximation

$$
\omega_{x}=K e^{-\gamma x}
$$

where $K$ and $\gamma$ are scaling constants.

For the present purpose it is essential, that each position $x$ along the basilar membrane is tuned to a specific frequency. The basic uncertainty principle contains the expectation value $\nu$ as a parameter. In the following this parameter will be identified with this specific frequency $\omega_{x}$. Assuming linearity and time invariance, the response of the inner ear to the input

$$
f(t)=R e e^{i \omega t}
$$

has to be of the form

$$
\operatorname{Re}\left\{\hat{g}(x, \omega) e^{i \omega t}\right\} .
$$

The variable $x$ denotes the position along the basilar membrane. The complex valued function $\hat{g}(x, \omega)$ is called the basilar membrane filter. It displays a remarkable invariance with respect to dilations:

$$
\hat{g}(x, \omega)=\hat{g}(x-\gamma \log a, a \omega), \quad a>0 .
$$

In this notation the highest audible frequency of about $20^{\prime} 000 \mathrm{~Hz}$ is assigned to the position $x=0$, the place at the oval window. The lower frequencies are assigned to places $x$ closer to the apex. The positive number $\gamma$ is the scaling constant that appears in the frequency position function.

This invariance property is amazingly exact for frequencies above $1000 \mathrm{~Hz}$ and changes slightly for lower frequencies. It is this invariance property that explains the dilation invariance of the hearing process. In her book I. Daubechies (1992) observes, that the ear produces a wavelet transform of the incoming sound. In the literature on acoustics this result appears in the work of X.Yang, K. Wang and S. Shamma (1992). The reason behind this fact is the invariance law for the basilar membrane filter.

In the present paper, the basic discovery is the fact, that the basilar membrane filter $\hat{g}(x, \omega)$ can be approximated by extremal functions for the general uncertainty inequality. With this in mind the extremal function $h$ for the uncertainty inequality is written in the form

$$
h(\omega)=c e^{-i \epsilon \operatorname{sign}(\omega)-i \beta \frac{\omega}{\nu}} e^{-\mu\left|\frac{\omega}{\nu}\right|}\left|\frac{\omega}{\nu}\right|^{\mu-\frac{1}{2}}
$$


with real constants $c, \varepsilon, \beta$ and $\mu$ that can be chosen arbitrarily in adjustment of the experimental data. The parameter $\nu$ takes the special role mentioned above. It is the expectation value of the extremal function $h$ and as such figures as a parameter in the uncertainty inequality. In the present context, it is identified with $\omega_{x}$. In this way, the extremal function $h$ becomes a function $h(x, \omega)$ of $x$ and $\omega$. It displays the scaling invariance

$$
h(x, \omega)=h(x-\gamma \log a, a \omega), \quad a>0 .
$$

This is the proposed representation for the basilar membrane filter. In Figure 1 the graph of this function is pictured as a function of the distance $x$ (in $\mathrm{mm}$ ) along the basilar membrane. It is reminiscent of Békésy's analysis of the dead human cochlea. (Békésy 1947). His results are described in Geisler's book (1998) in chapter 5 (compare in particular with Figures 5.2 and 5.3).

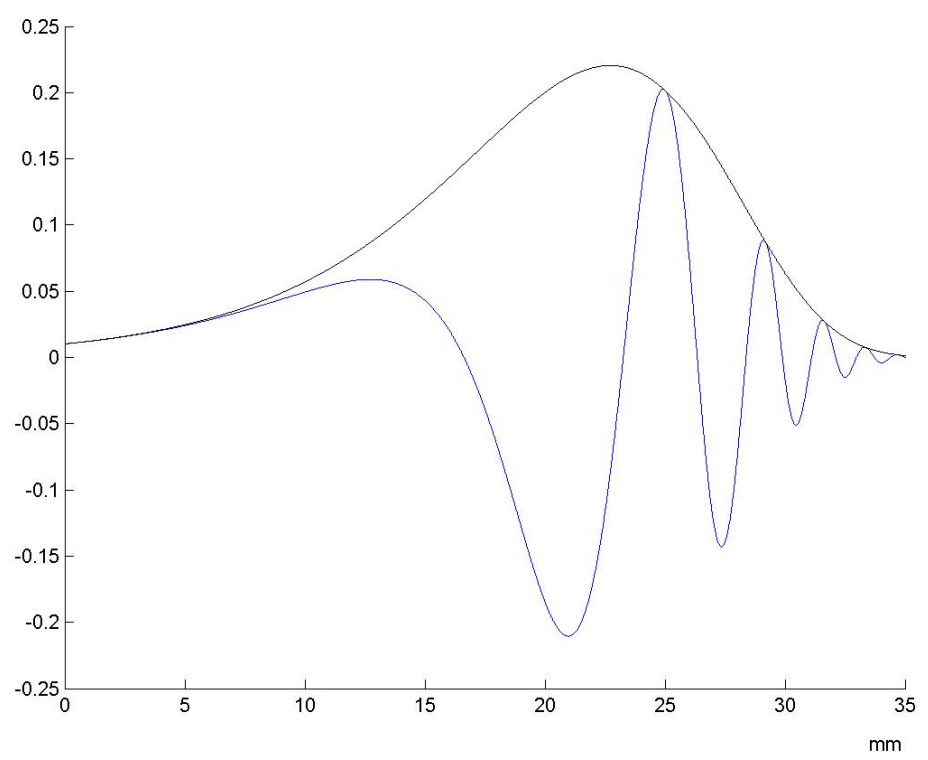

Figure 1: The real part and the modulus of the extremal function $h(x, \omega)$ as a function of $x$ for $\omega=314 \mathrm{~Hz}$. The parameters are: $c=1, \eta=1.6, \beta=2 \pi$

The linearity requirement in the definition of $\hat{g}(x, \omega)$ is essential. Unfortunately it only holds within narrow limits. The intensity of the acoustic signal varies over more than $100 \mathrm{~dB}$ whereas the basilar membrane movement varies over a much smaller range. The response of the inner ear is thus highly compressive, it can only be linear within a rather narrow range. The extremal function $h(x, \omega)$ is then interpreted as a linear approximation of the basilar membrane filter at high intensities of sound. In order to obtain a good linear approximation at low levels 
the uncertainty principle for the affine group will be generalized. This is the topic of the next section.

\section{The general uncertainty principle}

The mathematical construction starts with a real character $\chi$ of the multiplicative group $\mathbb{R}^{*}$

$$
\chi(a)=a^{\chi}, \quad a>0, \quad \chi>0
$$

and the quasiregular unitary representation of $\mathbb{R}^{*}$ on $L_{\mathrm{sym}}^{2}$ :

$$
\hat{\delta}^{\chi}(a) \hat{f}(\omega)=a^{\frac{1}{2 \chi}} \hat{f}\left(a^{\frac{1}{\chi}} \omega\right)
$$

To obtain a representation $\hat{\rho}^{\chi}$ of the affine group $\Gamma$ set

$$
\hat{\tau}^{\chi}(b) \hat{f}(\omega)=e^{-i b|\omega|^{\chi} \operatorname{sign}(\omega)} \hat{f}(\omega)
$$

and

$$
\hat{\rho}^{\chi}(a, b)=\hat{\tau}^{\chi}(b) \hat{\delta}^{\chi}(a)
$$

This is in fact a representation of $\Gamma$ on $L_{\mathrm{sym}}^{2}$ since

$$
\begin{aligned}
\hat{\rho}^{\chi}(a, b) \hat{\rho}^{\chi}\left(a^{\prime}, b^{\prime}\right) \hat{f}(\omega) & =e^{-i b|\omega|^{\chi} \operatorname{sign}(\omega)} a^{\frac{1}{2 \chi}} \hat{\rho}^{\kappa}\left(a^{\prime}, b^{\prime}\right) \hat{f}\left(a^{\frac{1}{\lambda}} \omega\right) \\
& =e^{-i b|\omega|^{\chi} \operatorname{sign}(\omega)} a^{\frac{1}{2 \chi}} e^{-i b^{\prime}\left|a^{\frac{1}{\chi}} \omega\right|^{\chi} \operatorname{sign}(\omega)} a^{\frac{1}{2 \chi}} \hat{f}\left(a^{\prime \frac{1}{\chi}} a^{\frac{1}{\chi}} \omega\right) \\
& =\hat{\rho}^{\chi}\left(a a^{\prime}, a b^{\prime}+b\right) \hat{f}(\omega)
\end{aligned}
$$

and it is clearly unitary.

The infinitesimal generators are

$$
\begin{aligned}
\hat{A}^{\chi} & =\frac{1}{\chi}\left(\frac{1}{2}+\omega \frac{d}{d \omega}\right)=\frac{1}{\chi} \hat{A} \\
\hat{B}^{\chi} & =-i \operatorname{sign}(\omega)|\omega|^{\chi}
\end{aligned}
$$

The commutator relation are those of the Lie algebra of $\Gamma$ :

$$
\left[\hat{A}^{\chi}, \hat{B}^{\chi}\right]=\hat{B}^{\chi}
$$

This representation is extended to a representation of $\Gamma \times S$ by setting

$$
\hat{\varepsilon}(\varphi) \hat{f}(\omega)=e^{-i \varphi \operatorname{sign}(\omega)} \hat{f}(\omega)
$$

The associated uncertainty inequality becomes

$$
\left|\left(\hat{f},\left[\hat{A}^{\chi}, \hat{H} \hat{B}^{\chi}\right] \hat{f}\right)\right| \leqslant 2\left\|\hat{A}^{\chi} \hat{f}\right\|\left\|\hat{H} \hat{B}^{\chi} \hat{f}\right\|
$$

The expectation $\nu_{\chi}$ of $-\hat{H} \hat{B}^{\chi}$ is defined as

$$
\nu_{\chi}\|\hat{f}\|^{2}=\left(\hat{f},-\hat{H} \hat{B}^{\chi} \hat{f}\right)=\int_{-\infty}^{\infty}|\omega|^{\chi}|\hat{f}(\omega)|^{2} d \omega .
$$


With $\hat{A}^{\chi}=\frac{1}{\chi} \hat{A}$ this leads to the inequality

$$
\nu_{\chi}\|\hat{f}\|^{2} \leqslant \frac{2}{\chi}\|\hat{A} \hat{f}\|\left\|\hat{H} \hat{B}^{\chi} \hat{f}\right\| .
$$

We arrived at almost the same inequality as before except for the fact, that the selfadjoint operator $-\hat{H} \hat{B}$ has been replaced by a power of $-\hat{H} \hat{B}$

$$
(-\hat{H} \hat{B})^{\chi}=-\hat{H} \hat{B}^{\chi}=|\omega|^{\chi} .
$$

Since $\hat{B}$ commutes with $\hat{H} \hat{B}^{\chi}$ the proof for the basic inequality can be modified to give the general uncertainty inequality

$$
\nu_{\chi}\|f\|^{2} \leqslant \frac{1}{\chi}\|(A-\beta B) f\|\left\|\left(H B^{\chi}-\eta I\right) f\right\| .
$$

The norms on the righthand side are minimal for

$$
\begin{aligned}
& \beta=\frac{1}{\|B f\|^{2}} \int t\left|\frac{d f}{d t}(t)\right|^{2} d t \\
& \eta=-\frac{1}{\|\hat{f}\|^{2}} \int|\omega|^{\chi}|\hat{f}(\omega)|^{2} d \omega:=-\nu_{\chi}
\end{aligned}
$$

The interpretation of the term $\|(\hat{A}-\beta \hat{B}) f\|$ is unchanged

$$
\begin{aligned}
\|(A-\beta B) f\|^{2} & =-\frac{1}{4}\|f\|^{2}+\int(t-\beta)^{2}\left|\frac{d f}{d t}(t)\right|^{2} d t \\
& =-\frac{1}{4}\|f\|^{2}+\sigma^{2}\|f\|^{2}
\end{aligned}
$$

with $\sigma^{2}$ the mean deviation of $\frac{d f}{d t}$ from its expected value $\beta$, normalized by $\|f\|^{2}$.

The remaining term is the mean deviation of $|\omega|^{\chi}$ from its expected value $\nu_{\chi}$ :

$$
\left\|\left(\hat{H} \hat{B}^{\chi}+\nu_{\chi} I\right) \hat{f}\right\|^{2}=\int\left(|\omega|^{\chi}-\nu_{\chi}\right)^{2}|\hat{f}(\omega)|^{2} d \omega:=\tau_{\chi}^{2}\|f\|^{2}
$$

Theorem 5.1 (general uncertainty principle). We have

$$
\frac{\chi^{2} \nu_{\chi}^{2}}{4} \leqslant\left(-\frac{1}{4}+\sigma^{2}\right) \tau_{\chi}^{2} .
$$

It is remarkable that this inequality transforms nicely under the standard representations $\rho$ (and $\hat{\rho}$ ), but not under the representation $\rho^{\chi}$. This is due to the fact that we used $A-\beta B$ and not $A-\beta B^{\chi}$.

Under the representation $\rho$ the quantities $\beta$ and $\sigma$ behave as before

$$
\begin{aligned}
& \beta\left(f_{a, b}\right)=a \beta+b, \\
& \sigma\left(f_{a, b}\right)=\sigma(f) .
\end{aligned}
$$


For $\nu_{\chi}$ and $\tau_{\chi}$ one obtains

$$
\begin{gathered}
\nu_{\chi}\left(f_{a, b}\right)=\frac{1}{\left\|\hat{f_{a, b}}\right\|^{2}} \int_{-\infty}^{\infty}|\omega| \chi\left|\hat{f_{a, b}}(\omega)\right|^{2} d \omega=a^{-\chi} \nu_{\chi}(f), \\
\tau_{\chi}\left(f_{a, b}\right)=\frac{1}{\left\|\hat{f_{a, b}}\right\|^{2}} \int_{-\infty}^{\infty}\left(|\omega|^{\chi}-\nu_{\chi}\left(\hat{f_{a, b}}\right)\right)^{2}\left|\hat{f_{a, b}}(\omega)\right|^{2} d \omega=a^{-2 \chi} \tau_{\chi}^{2}(f) .
\end{gathered}
$$

The Fourier transforms of the extremal functions for the general uncertainty inequality can be determined explicitly. They have to satisfy the equation

$$
\frac{\lambda}{\chi}\left(\left(\frac{1}{2}+\omega \frac{d}{d \omega}\right) \hat{f}+i \beta \omega \hat{f}\right)+\mu\left(-|\omega|^{\chi} \hat{f}-\nu_{\chi} \hat{f}\right)=0
$$

for certain real constants $\lambda, \mu, \beta$ and $\nu_{\chi}$, with $\nu_{\chi}>0$.

Set $\hat{f}=h, \frac{\mu \chi}{\lambda}=-\kappa$ and separate the equation:

$$
\frac{\frac{d h}{d \omega}}{h}=-\kappa \frac{|\omega|^{\chi}}{\omega}+\left(\kappa \nu_{\chi}-\frac{1}{2}\right) \frac{1}{\omega}-i \beta
$$

The solutions in $L_{\text {sym }}^{2}$ are

$$
h(\omega)=c e^{-i \varepsilon \operatorname{sign}(\omega)} e^{-\frac{\kappa}{\chi}|\omega|^{\chi}}|\omega|^{\kappa \nu_{\chi}-\frac{1}{2}} e^{-i \beta \omega}, \quad c, \varepsilon \in \mathbb{R}
$$

(with $\kappa>0, \nu_{\chi}>0$ ).

Again, $\kappa$ can be determined from the defining equality

$$
\begin{aligned}
\|(A-\beta B) h\|^{2} & =\kappa^{2}\left\|\left(H B^{\chi}+\nu_{\chi}\right) h\right\|^{2}, \\
-\frac{1}{4}+\sigma^{2} & =\kappa^{2} \tau_{\chi}^{2} .
\end{aligned}
$$

Since $h$ is extremal

$$
\frac{\chi^{2} \nu_{\chi}^{2}}{4 \tau_{\chi}^{2}}=-\frac{1}{4}+\sigma^{2}
$$

Therefore

$$
\kappa=\frac{\chi \nu_{\chi}}{2 \tau_{\chi}^{2}}=\frac{2}{\chi \nu_{\chi}}\left(-\frac{1}{4}+\sigma^{2}\right) .
$$

Proposition 5.1. The set $E_{\chi}$ of extremal functions for the general uncertainty inequality that are in $L^{2}(\mathbb{R}, \mathbb{R})$ is invariant under the action $\rho$ of $\Gamma \times S$.

Proof. It suffices to show that the set $\hat{E}_{\chi}$ of Fourier transforms of extremal functions is invariant under $\hat{\rho}$. It clearly is invariant under $\hat{\varepsilon}$. The invariance under $\hat{\tau}$ is due to the factor $e^{-i \beta \omega}$ and the invariance under $\hat{\delta}$ can be verified directly.

$$
\hat{\delta}(a) h(\omega)=a^{\kappa \nu_{\chi}-\frac{1}{2}} c e^{-i \varepsilon \operatorname{sign}(\omega)} e^{\frac{\kappa a \chi}{\chi}|\omega|^{\chi}}|\omega|^{\kappa \nu_{\chi}-\frac{1}{2}} e^{-i \beta a \omega} .
$$


A

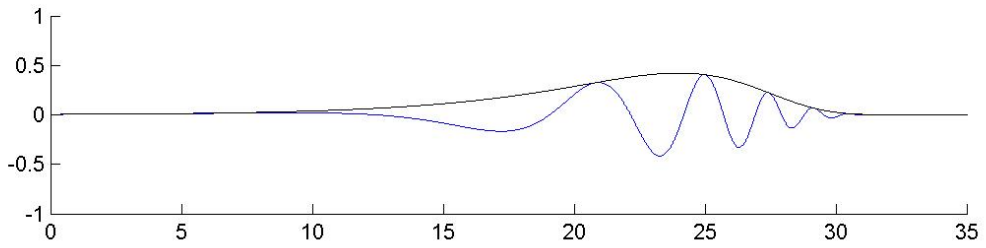

B

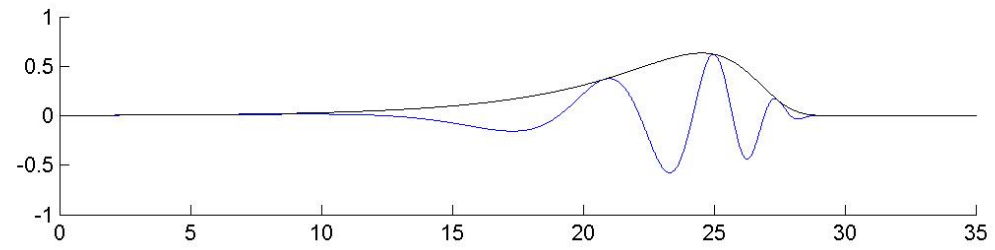

$\mathrm{C}$

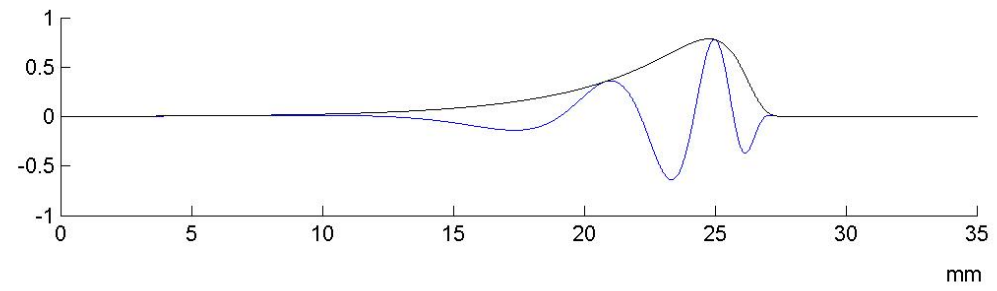

Figure 2: Real part and modulus of the extremal functions $h_{\chi}(x, \omega)$ for $\omega=314 \mathrm{~Hz}$. A: $\chi=2, \mathrm{~B}: \chi=4$ and $\mathrm{C}: \chi=8$. The remaining parameters are: $\eta=4 \pi, \mu=2, C=1$ and $\varepsilon=0$.

The localization parameter for our application is

$$
\nu=\left(\nu_{\chi}\right)^{\frac{1}{\chi}}
$$

Setting

$$
\mu=\frac{\kappa \nu_{\chi}}{\chi}, \quad \eta=\frac{\beta}{\nu}
$$

the extremal function can then be expressed in the form

$$
h_{\chi}(\omega)=c e^{-\varepsilon \operatorname{sign}(\omega)-i \eta \frac{\omega}{\nu}} e^{-\mu\left|\frac{\omega}{\nu}\right|^{\chi}}\left|\frac{\omega}{\nu}\right|^{\mu \chi-\frac{1}{2}}
$$

with newly defined constants c and $\varepsilon$. Once again, setting $\nu=\omega_{x}$ turns $h_{\chi}$ into a function $h_{\chi}(x, \omega)$ of $x$ and $\omega$ that displays the correct scaling behavior:

$$
h_{\chi}(x, \omega)=h_{\chi}(x-\gamma \log a, a \omega), \quad a>0 .
$$

The function $h_{\chi}(x, \omega)$ is our approximation for $\hat{g}(x, \omega)$ at small intensities, the level decreasing with increasing values of $\chi$. The functions $h_{\chi}$ for $\chi=2,4$ and 8 are illustrated in Figure 2. 
The maximal value of the modulus of the extremal function $\left|h_{\chi}(\omega)\right|=$ $|c| e^{-\mu\left|\frac{\omega}{\nu}\right|^{\chi}}\left|\frac{\omega}{\nu}\right|^{\mu \chi-\frac{1}{2}}$ is reached at a value

$$
\omega^{*}=\nu\left(1-\frac{1}{2 \mu \chi}\right)^{\frac{1}{\chi}}
$$

below $\nu=\omega_{x}$. This agrees with the observation, that at a fixed position $x$ the peak of the basilar membrane filter $|g(x, \omega)|$ moves to lower frequencies with increasing sound levels. If the parameter $\chi$ increases then the ratio $\frac{\nu}{\omega^{*}}$ tends to 1 . This is illustrated in Figure 3, in which the graphs for $\left|h_{\chi}(\omega)\right|$ are shown in a log-log scale.

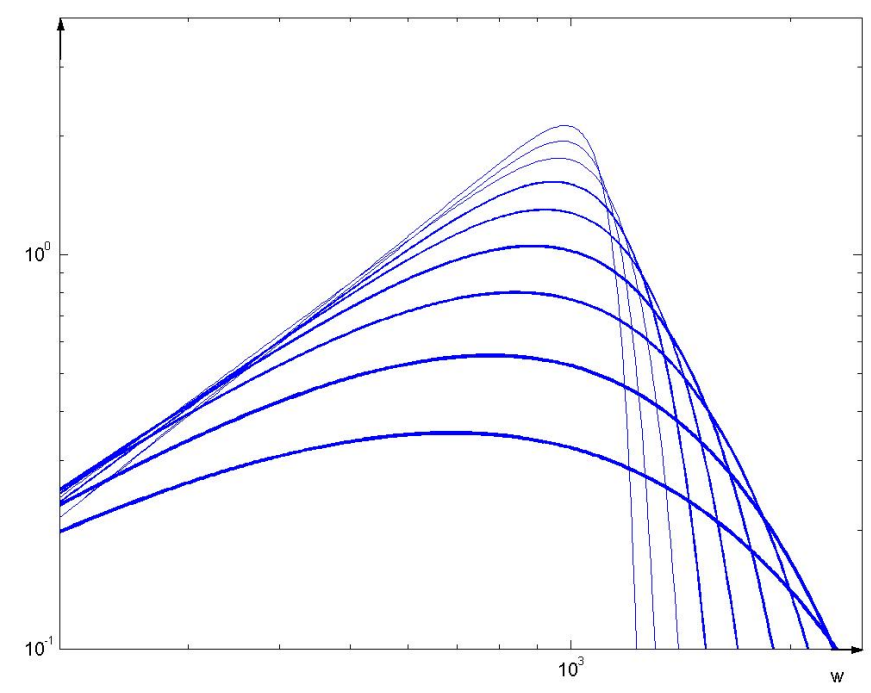

Figure 3: The extremal functions $\left|h_{\chi}(\omega)\right|$ on a $\log \log$-scale for $\nu=1000, \mu$ from 1.6 to 2 ; c from 1.6 to 2.4 and successively for $\chi=1,1.41,2,2.82,4,5.64,8,11.28$ and 16 (decreasing width of line)

\section{Intertwining}

Proposition 6.1. The unitary operator $T: L^{2}(\mathbb{R}) \rightarrow L^{2}(\mathbb{R})$,

$$
T h(\omega)=\sqrt{\chi}|\omega|^{\frac{\chi-1}{2}} h\left(|\omega|^{\chi} \operatorname{sign}(\omega)\right)
$$

intertwines the representations $\hat{\rho}$ and $\hat{\rho}^{\chi}$

$$
\hat{\rho}^{\chi} T=T \hat{\rho} .
$$

Furthermore, it commutes with the action of $S$. 
Proof. $T$ is unitary:

$$
\begin{aligned}
\|T h\|^{2} & =\chi \int_{-\infty}^{\infty}|\omega|^{\chi-1}\left|h\left(|\omega|^{\chi} \operatorname{sign}(\omega)\right)\right|^{2} d \omega \\
& =\int|h(\mu)|^{2} d \mu=\|h\|^{2} .
\end{aligned}
$$

The intertwining property is easily verified:

$$
\begin{aligned}
\hat{\rho}_{(a, b)}^{\chi} T h(\omega) & =\hat{\tau^{\chi}}(b) \hat{\delta}^{\chi}(a) T h(\omega) \\
& =e^{-i b|\omega|^{\chi}} \operatorname{sign}(\omega) a^{\frac{1}{2 \chi}} \sqrt{\chi}\left|a^{\frac{1}{\chi}} \omega\right|^{\frac{\chi-1}{2}} h\left(a|\omega|^{\chi} \operatorname{sign}(\omega)\right) \\
& =T \hat{\rho} h(\omega)
\end{aligned}
$$

The operator $T$ then also intertwines the infinitesimal operators

$$
\begin{aligned}
& \hat{A}^{\chi} T=T \hat{A}, \\
& \hat{B}^{\chi} T=T \hat{B}, \\
& \hat{H} T=T \hat{H} .
\end{aligned}
$$

The uncertainty principle in its basic form (Section 2) can be intertwined to give

$$
\left|\left(f, H B^{\chi} f\right)\right| \leqslant 2\left\|\left(A^{\chi}-\beta_{\chi} B^{\chi}\right) f\right\|\left\|\left(H B^{\chi}+\nu_{\chi}\right) f\right\| .
$$

The quantities $\nu_{\chi}$ and $\tau_{\chi}$ are as before, and

$$
\beta_{\chi}=\frac{\operatorname{Re}\left(A^{\chi} f, B^{\chi} f\right)}{\left\|B^{\chi} f\right\|}
$$

If $\chi=n \in \mathbb{N}$, then $\beta_{\chi}$ can be expressed as an integral in the time variable.

If $n=2 m+1$ is odd, then

$$
\begin{aligned}
\hat{B} & =-|\omega|^{n} i \operatorname{sign}(\omega)=(-1)^{m+1}(i \omega)^{n}, \\
n \operatorname{Re}\left(A^{n} f, B^{n} f\right) & =(-1)^{m} \operatorname{Re} \int\left(\frac{1}{2} f+t \frac{d}{d t} f\right) \frac{d^{n}}{d t^{n}} \bar{f} d t \\
& =(-1)^{m-1} \operatorname{Re} \int\left(\frac{1}{2} \frac{d}{d t} f+t \frac{d^{2}}{d t} f\right) \frac{d^{n-1}}{d t^{n-1}} \bar{f} d t \\
& =n \operatorname{Re}\left(A^{n} \frac{d f}{d t}, B^{n-2} \frac{d f}{d t}\right)=\operatorname{Re}\left(A \frac{d^{m} f}{d t^{m}}, B \frac{d^{m} f}{d t^{m}}\right) \\
& =\int t\left|\frac{d^{m+1} f}{d t^{m+1}}\right|^{2} d t=n \beta_{n}\left\|B^{n} f\right\|^{2}
\end{aligned}
$$


If $n=2 m$ is even, then

$$
\begin{aligned}
\hat{B}^{n} & =(-1)^{m+1}(i \omega)^{2 m} i \operatorname{sign}(\omega), \\
n \operatorname{Re}\left(A^{n} f, B^{n} f\right) & =(-1)^{m} \operatorname{Re} \int(A f) H \frac{d^{n} \bar{f}}{d t^{n}} d t \\
& =(-1)^{m-1} \operatorname{Re} \int\left(A \frac{d f}{d t}\right) H \frac{d^{n-1} \bar{f}}{d t^{n}} d t \\
& =n \operatorname{Re}\left(A^{n} \frac{d f}{d t}, B^{n-2} \frac{d f}{d t}\right)=n \operatorname{Re}\left(A^{n} \frac{d^{m} f}{d t^{m}}, H \frac{d^{m} f}{d t^{m}}\right) \\
& =\operatorname{Re} \int t \frac{d^{m+1} f}{d t^{m+1}} H \frac{d^{m} \bar{f}}{d t^{m}} d t=n \beta_{n}\left\|B^{n} f\right\|^{2}
\end{aligned}
$$

For both $n$ even and odd

$$
\left\|B^{n} f\right\|^{2}=\int\left|\frac{d^{n}}{d t^{n}} f(t)\right|^{2} d t
$$

The quantity $\left\|\left(A^{n}-\beta_{n} B^{n}\right) f\right\|^{2}$ can be calculated to be

$$
-\frac{1}{4 n^{2}}\|f\|^{2}+\int\left|\frac{t}{n} \frac{d f}{d t}-(-1)^{m} \beta_{n} \frac{d^{n} f}{d t^{n}}\right|^{2} d t:=\frac{1}{n^{2}}\left(-\frac{1}{4}+\sigma_{n}^{2}\right)
$$

in case $n=2 m+1$, and finally for $n=2 m$ :

$$
-\frac{1}{4 n^{2}}\|f\|^{2}+\int\left|\frac{t}{n} \frac{d f}{d t}+(-1)^{m} \beta_{n} H \frac{d^{n} f}{d t^{n}}\right|^{2} d t:=\frac{1}{n^{2}}\left(-\frac{1}{4}+\sigma_{n}^{2}\right)
$$

Theorem 6.1 (general uncertainty principle, second version). We have

$$
\frac{n^{2} \nu_{n}^{2}}{4} \leqslant\left(-\frac{1}{4}+\sigma_{n}^{2}\right) \tau_{n}^{2}
$$

For $n=1$ this is the basic uncertainty principle for which we calculated the extremal functions

$$
h(\omega)=c e^{-i \varepsilon \operatorname{sign}(\omega)} e^{-\kappa|\omega|-i \beta \omega}|\omega|^{\kappa \nu-\frac{1}{2}}
$$

in Section 2. The extremal functions for general $n$ are then obtained by applying the intertwining operator $\mathrm{T}$.

If we choose $\beta=0$, then

$$
T h(\omega)=\sqrt{n} c e^{-i \varepsilon \operatorname{sign}(\omega)}|\omega|^{\frac{n-1}{2}} e^{-\kappa|\omega|^{n}}|\omega|^{n \kappa \nu-\frac{1}{2}}
$$

for both versions of the general uncertainty principle. The set $E_{\chi}$ of extremal functions for the second version of the general uncertainty inequality is invariant under the action $\rho^{\chi}$ of $\Gamma \times S$. 


\section{Heisenberg's uncertainty principle for real valued functions}

The classical Heisenberg uncertainty principle tells us that position and momentum of a particle cannot be determined simultaneously with arbitrary precision. In quantum mechanics the particles are represented by complex valued functions. The expectation for the momentum is defined in the Fourier domain as

$$
\int_{\mathbb{R}} \omega|\hat{f}|^{2} d \omega
$$

(with normalization $\|f\|=1$ ). Yet this definition assigns the value zero to all real valued functions. This fact lies at the core of the difficulties encountered in applying the Heisenberg inequality in signal processing. However there is a way to formulate the Heisenberg uncertainty principle that is much more appropriate for real valued functions.

Powers of the selfadjoint operator $-\hat{H} \hat{B}$ lead to the general uncertainty principle for $\Gamma \times S$. Other functions of $-\hat{H} \hat{B}$ may also lead to interesting inequalities. The example which is chosen here is the logarithm.

Since $-\hat{H} \hat{B}$ is a positive selfadjoint operator, $\log (-\hat{H} \hat{B})$ is a well defined selfadjoint operator. In $L_{\text {sym }}^{2}$ it is realized as the multiplier $\log |\omega|$. It commutes with $\hat{B}$ and $\hat{H}$ and the commutator relation with $\hat{A}$ is the Heisenberg relation:

$$
[\hat{A}, \log (-\hat{H} \hat{B})]=I
$$

The uncertainty inequality is derived in the same way:

$$
\|f\|^{2} \leqslant 2\|(A-\beta B) f\|\|(\log (-H B)-\nu I) f\| .
$$

The values $\beta$ and $\eta$ for which the norms of the righthand side are minimal are the expectation values

$$
\beta=\frac{1}{\left\|\frac{d f}{d t}\right\|^{2}} \int_{-\infty}^{\infty} t\left|\frac{d f}{d t}(t)\right|^{2} d t
$$

and

$$
\nu=\frac{1}{\|f\|^{2}} \int_{-\infty}^{\infty} \log |\omega||\hat{f}(\omega)|^{2} d \omega
$$

As before we define $\sigma$ by

$$
\sigma^{2}\|f\|^{2}=\int_{-\infty}^{\infty}(t-\beta)^{2}\left|\frac{d f}{d t}(t)\right|^{2} d t
$$

and $\tau_{\log }^{2}$ as the mean deviation

$$
\tau_{\log }^{2}\|f\|^{2}=\int_{-\infty}^{\infty}(\log |\omega|-\nu)^{2}|\hat{f}(\omega)|^{2} d \omega
$$




\section{Theorem 7.1 (Heisenberg's uncertainty principle for real valued func-} tions). We have

$$
\frac{1}{4} \leqslant\left(-\frac{1}{4}+\sigma^{2}\right) \tau_{\log }^{2} .
$$

The extremal functions are determined by the differential equation

$$
\lambda\left(\frac{1}{2}+\omega \frac{d}{d \omega} \hat{f}+i \beta \omega \hat{f}\right)+\mu(\log |\omega| \hat{f}-\nu \hat{f})=0
$$

Again we set $\hat{f}=h, \frac{\mu}{\lambda}=\kappa$ and separate the equation

$$
\frac{\frac{d h}{d \omega}}{h}=-i \beta+\left(\kappa \nu-\frac{1}{2}\right) \frac{1}{\omega}-\kappa \frac{\log |\omega|}{\omega}
$$

Integration gives

$$
\begin{aligned}
\log |h(\omega)| & =-i \beta \omega+\left(\kappa \nu-\frac{1}{2}\right) \log |\omega|-\kappa \frac{(\log |\omega|)^{2}}{2}+\text { const } \\
h(\omega) & =c e^{-i \varepsilon \operatorname{sign}(\omega)-i \beta \omega} e^{-\frac{\kappa}{2}(\log |\omega|)^{2}}|\omega|^{\kappa \nu-\frac{1}{2}}
\end{aligned}
$$

with arbitrary real constants $c$ and $\varepsilon, \beta=\beta(h), \nu=\nu(h)$ and $\kappa=\frac{1}{2 \tau^{2}}=$ $2\left(-\frac{1}{\mu}+\sigma^{2}\right)$.

This function is in $L_{\text {sym }}^{2}$ if $\kappa>0$ (this time $\nu$ is an arbitrary real number).

Proposition 7.1. The set of extremal functions that are in $L_{\mathrm{sym}}^{2}$ is invariant under the action $\rho$ of $\Gamma \times S$.

Remark. With the substitution $x=\log \omega,(\omega>0)$ the function

$$
\tilde{f}(x)=h(\omega)=c e^{-i \varepsilon-i \beta e^{x}} e^{-\frac{\kappa x^{2}}{2}+\left(\kappa \nu-\frac{1}{2}\right) x}
$$

is almost the extremal function for the well known Heisenberg inequality as it is usually derived for the standard action of the Heisenberg group on the complex Hilbert space $L^{2}(\mathbb{R}, \mathbb{C})$.

In order to arrive at the classical solution one has to replace $A$ by $A-\alpha H$ (and not $A-\beta B)$ in the uncertainty inequality

$$
1 \leqslant 2\|A f\|\|\log (-H B) f\| .
$$

This gives

$$
1 \leqslant 2\|(A-\alpha H) f\|\|(\log (-H B)-\nu I) f\|
$$

where the norms are minimal for

$$
\alpha=\frac{(A f, H f)}{\|f\|^{2}}
$$


and for the expectation value

$$
\nu=\frac{1}{\|f\|^{2}} \int \log |\omega||f(\omega)|^{2} d \omega .
$$

The differential equation for the extremal function is

$$
\lambda\left(\left(\frac{1}{2}+\omega \frac{d}{d \omega}\right) \hat{f}+i \alpha \operatorname{sign}(\omega) \hat{f}\right)+\mu(\log |\omega| \hat{f}-\nu \hat{f})=0 .
$$

Set $\hat{f}=h, \frac{\mu}{\lambda}=\kappa$ and separate the equation

$$
\frac{\frac{d h}{d \omega}}{h}=-\kappa \frac{\log |\omega|}{\omega}+\left(\kappa \nu-\frac{1}{2}\right) \frac{1}{\omega}-i \alpha \frac{1}{|\omega|} .
$$

The solutions are

$$
h(\omega)=c e^{-i \varepsilon \operatorname{sign}(\omega)} e^{-\kappa\left(\frac{\log |\omega|}{2}\right)^{2}}|\omega|^{\kappa \nu-\frac{1}{2}}|\omega|^{-i \alpha \operatorname{sign}(\omega)} .
$$

The substitution $x=\log \omega$ (for $\omega>0$ ) gives the classical functions

$$
\tilde{h}(x)=h(\omega)=c e^{-i \varepsilon} e^{-\kappa \frac{x^{2}}{2}+\left(\kappa \nu-\frac{1}{2}-i \alpha\right) x} .
$$

\section{References}

[C] L. Cohen, The scale representation, IEEE Transactionson Signal Processing 41 (1993), 3275-3292.

[D] I. Daubechies, Ten lectures on wavelets, SIAM, Philadelphia, 1992.

[FS] G. Folland, A. Sitaram, The uncertaintyprinciple: a mathematical survey, J. Fourier Anal. Appl. 3 (1997), 207-238.

[G] D. Gabor, Theory of communication, J. IEE 93 (1946), 429-457.

[H] H. Helmholtz, On the Sensation of Tone, English translation of the 4th edition by A.J. Ellis, London, 1912.

[IP] T. Irino, R. D. Patterson, A time-domain, level-dependent auditory filter: Thegammachirp, J. Acoust. Soc. Am. 101 (1997), 412-419.

[M] S. Mallat, A wavelet tour of signal processing, Adademic Press, San Diego, 1998.

[P] R. D. Patterson, The sound of a sinusoid: Spectral models, J. Acoust. Soc. Am. 96 (1994), 1409-1418.

[R] H. M. Reimann, Invariance principles for cochlear mechanics: Hearing phases, J. Acoust. Soc. Am. 119 (2006), 997-1004.

[S] E. M. Stein, Singular integrals ans differentiability properties of functions, Princeton University Press, 1970

[T] M. E. Taylor, Noncommutative harmonic analysis, AMS, Providence, 1986.

[V] G. Von Békésy, The variation of phase along the basilar membrane with sinusoidal vibraitions, J. Acoust. Soc. Am. 19 (1947), 452-460. 
[YWS] X. Yang, K. Wang, S. Shamma, Auditory representation ofacoustic signals, IEEE Transaction on Information theory 38 (1992), 824-839.

Address: H.M. Reimann: University of Berne, Institute of Mathematics, Sidlerstrass 5, 3012 Berne, Switzerland.

E-mail: reimann@math.unibe.ch

Received: 31 March 2008; revised: 13 November 2008 
